\title{
CONSIDERACIONES SOBRE AMPLIFICACIÓN SÍSMICA EN DEPÓSITOS ALUVIALES, CASO CUENCA DEL TEMPISQUE, COSTA RICA
}

\author{
Martín Rojas \\ Instituto Costarricense de Electricidad \\ E-mail: MRojasB @ice.go.cr
}

(Recibido: 7/1/00; Aceptado: 14/7/03)

\begin{abstract}
The study and analysis of seismic amplification was developed from the geologic setting of the Tempisque Basin and other regions with similar geological settings (alluvial deposits considerate as soft soils). The first well group (northwestern of the basin) gave maximum acceleration's amplitudes between 0,55-0,91 sec for an oscillation period of soil approximately of 0,88 and $0,71 \mathrm{sec}$. This area is where the biggest thicknesses of soft soil are located (between 35-60 m). The second group (central part) gave a period range for the maximum amplitude of 0,24-0,85 sec for an oscillation period approximate in 0,65 and 0,54 sec. The soft soil thicknesses are intermediate between $30-50 \mathrm{~m}$. The third group (middle and southeastern part) present values of maximum amplitude in the range between $0,10-0,55 \mathrm{sec}$ for an oscillation period of 0,23 and $0,14 \mathrm{sec}$. The thicknesses are minimum with values between $10-20 \mathrm{~m}$. The spectral values of amplification in the rank considerated are $0,5-$ $1,1 \mathrm{~g}, 0,55-0,98 \mathrm{~g}$ and $0,4-1,5 \mathrm{~g}$ for the first, second and third well group respectively. For the third group the amplification factors trend to overestimate the maximum values of amplification for about $2 \mathrm{~g}$.

The classification of soil types according to FEMA and ATC's discernments was done in four types: B, C, D and E (rock, soft rock and very dense soil, soft soil, very soft soil).
\end{abstract}

RESUMEN: El estudio y análisis de amplificación sísmica se desarrollaron a partir de la información geológica de la cuenca del Tempisque correlacionado con otras regiones de condiciones geológicas semejantes (rellenos aluviales caracterizados como suelos blandos).

Para el primer grupo de pozos (noroeste de la cuenca), los resultados en el ámbito de las aceleraciones espectrales en los períodos de máxima amplitud se obtienen en un rango de períodos dado entre 0,55-0,91 s para un período fundamental de oscilación del suelo aproximado en 0,88 y 0,71 s. En esta área se ubican los mayores espesores de suelo blando $(35-60 \mathrm{~m})$. En el segundo grupo (parte central) los períodos de máxima amplitud se dan en un rango de 0,24-0,85 s para un período de oscilación aproximado en 0,65 y 0,54 s. Los espesores de suelo blando presentan valores intermedios entre los 30-50 m. El tercer grupo (parte central y sureste) presenta valores de máxima amplitud en el rango de 0,10-0,55 s para un período de oscilación en 0.23 y 0.14 s. Los espesores de suelo blando son mínimos con valores de 10-20 m. Los valores espectrales de amplificación en los rangos de períodos determinados se dan entre $0,5-1,1 \mathrm{~g}, 0,55-0,98 \mathrm{~g}$ y $0,4-1,5 \mathrm{~g}$ para el primer, segundo y tercer grupo de pozos respectivamente. Los factores de amplificación para el caso del tercer grupo, tienden a sobrestimar los valores de amplificación cercanos a $2 \mathrm{~g}$.

La clasificación de tipos de suelos según los criterios de la FEMA y ATC se hizo en cuatro tipos: B, C, D y E (roca, suelo muy denso y roca blanda, suelo blando, suelo muy blando). 


\section{INTRODUCCIÓN}

El área de estudio abarca la parte central y norte de la península de Nicoya, representada en su mayor parte por la cuenca del Tempisque (Fig.1). Se obtuvo la información de 17 pozos hidrogeológicos, con sus respectivos perfiles geológicos y registros de resistividad eléctrica, distribuidos en tres grupos a lo largo de la cuenca del Tempisque. Se elaboró un modelo de capas geológicas para cada pozo con sus respectivos datos geofísicos. Esta información se procesó en el programa ProShake (1998) para obtener los resultados de amplificación. Se utilizaron los acelerogramas de los sismos de Limón del 22 de abril de 1991, registrado en roca en la estación Cachí y el sismo de Loma Prieta del 17 de octubre de 1989, registrado en roca en la estación de la Isla Yerva Buena sobre la Formación Franciscano (California, EE.UU.). El primer grupo de pozos se ubica al noroeste, comprendiendo las hojas Carrillo Norte y Belén. El segundo grupo se localiza al sureste de la hoja Belén en la parte central. El tercer grupo se ubica en la parte central y sureste, comprendiendo las hojas Tempisque, Talolinga y Matambú (Fig. 1 y Cuadro 1).

La cuenca del Tempisque es considerada como un sinclinal o graben, cuya estructura ha sido el resultado de una historia tectónica muy complicada. Estas características han sido propicias para la depositación de un espesor considerable de materiales erosionados de las formaciones geológicas adyacentes considerados, como suelos blandos, que en algunas partes alcanza hasta los $70 \mathrm{~m}$ de espesor.

Se ha determinado que la amplificación espectral en suelos es mucho mayor para depósitos no cohesivos profundos (arenas) y para depósitos de arcillas blandas a medianamente firmes que para condiciones de suelo firme o roca (Figs. 2 y 3). Lo anterior se puede resumir diciendo que depósitos de suelos blandos y profundos introducen una proporción significativamente mayor de componentes de período largo, en los movimientos de la superficie del terreno, los efectos aparentemente se incrementan con el aumento del espesor o blandura del suelo.
Las condiciones de sitio local tienen mucha importancia en todas las características del movimiento fuerte del terreno, como lo son: amplitud, contenido de frecuencias y duración. La extensión de esta influencia depende de la geometría y propiedades del material del subsuelo, de la topografía del sitio y de las características del movimiento sísmico (Seed \& Idriss, 1982).

Actualmente los efectos de sitio han sido evaluados por diferentes métodos: análisis teórico de respuesta del terreno (mediante instrumentación midiendo los movimientos del terreno en algún sitio determinado) o, mediante mediciones del movimiento de la superficie del terreno en sitios con diferentes condiciones de subsuelo. La base para un estudio de amplificación puede ser ilustrada analíticamente usando un análisis teórico simplificado de respuesta sísmica del terreno, como el que utiliza el programa SHAKE. Sin embargo, el principio básico y el modelo matemático que utiliza este programa idealiza algunas de las condiciones reales que se han determinado. El modelo de suelo que propone el programa es una sucesión de capas horizontales semiinfinitas, sobre un semi-espacio elástico de profundidad infinita. La perturbación o excitación

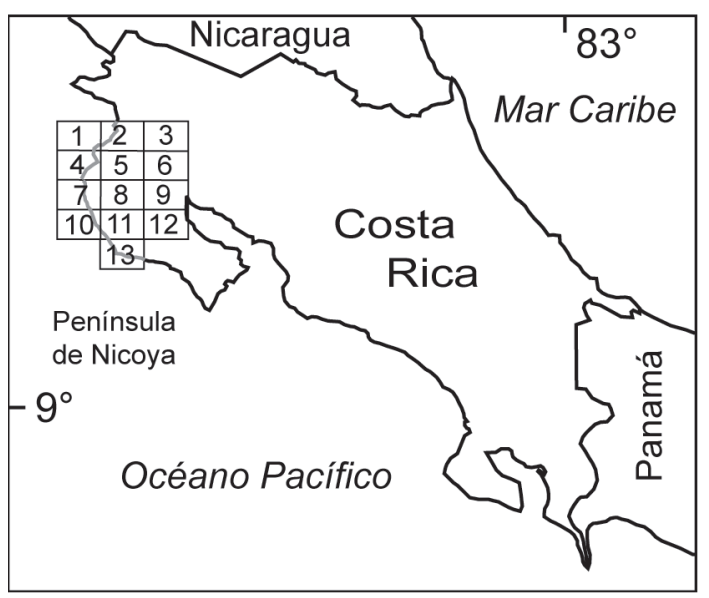

Fig. 1: Mapa de ubicación de las hojas cartográficas que incluye el área de estudio 1. Punta Gorda; 2. Carrillo Norte; 3. Monteverde; 4. Matapalo; 5. Belén; 6. Tempisque; 7. Villarreal; 8. Diriá; 9. Talolinga; 10. Marbella; 11. Cerro Brujo; 12. Matambú; 13. Garza. Editadas por el I.G.N. San José a escala 1:50 000 . 
Cuadro 1

Períodos de amplificación y oscilación del suelo

\begin{tabular}{|c|c|c|c|c|c|c|c|c|}
\hline \multicolumn{3}{|c|}{ Grupo 1} & \multicolumn{2}{|c|}{ Grupo 2} & \multicolumn{4}{|c|}{ Grupo 3} \\
\hline \multirow{2}{*}{$\begin{array}{l}\text { Pozos } \\
\text { Sismos }\end{array}$} & \multicolumn{2}{|c|}{$\begin{array}{l}\mathrm{CN}-33,71,72,73, \\
\quad 77,129,44\end{array}$} & \multicolumn{2}{|c|}{ BE-69, 105, 136} & \multicolumn{2}{|c|}{$\begin{array}{c}\text { A } \\
\text { TE9, TAL126, MT76 }\end{array}$} & \multicolumn{2}{|c|}{$\frac{\text { B }}{\text { TE14, TAL127, MT75 }}$} \\
\hline & Limón & Loma Prieta & Limón & Loma Prieta & Limón & Loma Prieta & Limón & Loma Prieta \\
\hline Espectral (seg) & 0,85 & 0,65 & 0,55 & 0,45 & 0,24 & 0,26 & 0,14 & 0,12 \\
\hline F.T. (seg) & 0,91 & 0,77 & 0,75 & 0,63 & 0,21 & 0,19 & 0,15 & 0,14 \\
\hline F.A. & 4,74 & 6,43 & 3,38 & 4,08 & 8,45 & 12,1 & 10,25 & 14,82 \\
\hline Espesor & \multicolumn{2}{|c|}{$35-60 m$} & \multicolumn{2}{|c|}{$30-50 \mathrm{~m}$} & \multicolumn{4}{|c|}{$10-20 m$} \\
\hline Rango (seg) & \multicolumn{2}{|c|}{$0,55-0,91$} & \multicolumn{2}{|c|}{$0,24-0,85$} & \multicolumn{4}{|c|}{$0,10-0,55$} \\
\hline Asignado (seg) & \multicolumn{2}{|c|}{$0,88 / 0,71$} & \multicolumn{2}{|c|}{$0,65 / 0,54$} & \multicolumn{2}{|c|}{0,23} & \multicolumn{2}{|c|}{0,14} \\
\hline
\end{tabular}

F.T.: Función de transferencia, F.A.: Factor de amplificación, CN: Hoja Carrillo Norte, BE: Hoja Belén, TE: Hoja Tempisque, TAL: Hoja Talolinga, MT: Hoja Matambú. Ubicación en figuras 1 y 4.

de las capas debido a un sismo se considera debido al efecto de la propagación de ondas cortantes que se propagan verticalmente desde la base rocosa del depósito hasta la superficie, pero sin considerar el efecto de las ondas superficiales Rayleigh y Love.

En Costa Rica se han desarrollado estudios de amplificación sísmica, sobre todo en el Valle Central: Gallegos (1980); Valverde (1981); Pujol \& Castro (1981); Vargas (1987); Taylor (1994); Ramírez et al. (1994); Ramírez (1995). El área de la península de Nicoya y la cuenca del Tempisque presenta características de alta sismicidad y grandes rellenos aluviales que la clasifican como una región de condiciones potenciales para la amplificación sísmica.

\section{ANÁLISIS DE LA INFORMACIÓN}

\section{Tipos de suelos}

Se definieron cuatro tipos de suelos, basado en el tipo de roca madre presente y los valores de velocidad de onda cortante y resistividad eléctrica, de acuerdo con información geofísica de SENARA (1997).
Según los criterios de la FEMA (Federal Emergency Management Agency, 1994) y la ATC (Aplied Technology Council, 1985), los suelos se clasifican en B, C, D y E (Fig. 4):

Tipo B (roca): corresponde con las rocas del Complejo de Nicoya: basaltos, intrusivos (gabros, dioritas, plagiogranitos) y radiolaritas. Según los datos reportados en informes técnicos, las velocidades de onda cortante en el basamento oscilan entre los $1500-2800 \mathrm{~m} / \mathrm{s}$. Tomando en cuenta el grado de meteorización del basamento

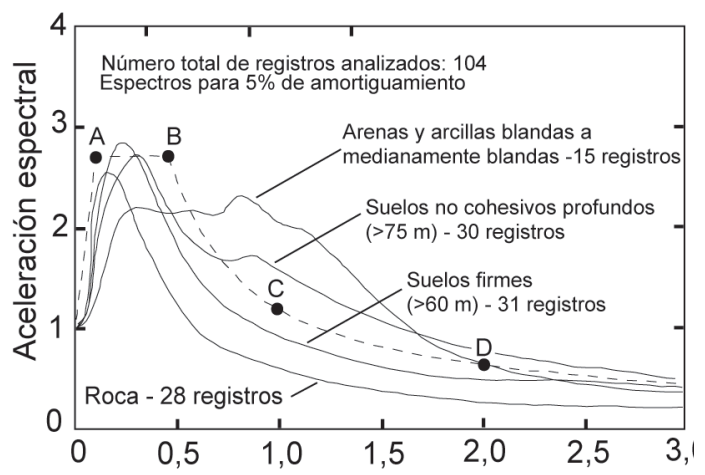

Fig. 2: Espectros de aceleración promedio para diferentes condiciones locales (Seed \& Idriss, 1982). 
en superficie, se deduce que tienen velocidades mayores a los $760 \mathrm{~m} / \mathrm{s}$ para una clasificación Tipo B (FEMA, 1994). De acuerdo con la ATC (1985), se clasifica como tipo S1: Roca. Los valores de resistividad varían entre los 50 - 250 ohmios-metro dependiendo del grado de fracturación, presencia de agua y sales minerales en la matriz, y el grado de meteorización de la roca.

Tipo C (suelo muy denso y roca blanda): corresponde con los suelos originados de las rocas sedimentarias: calcilutitas, radiolaritas, calizas, lutitas calcáreas y silíceas, areniscas, calizas arrecifales. También se incluyen en esta clasificación los suelos originados por las rocas piroclásticas de las formaciones Bagaces y Liberia: tobas e ignimbritas. Se deduce que los valores en las velocidades de onda cortante son superiores a los $360 \mathrm{~m} / \mathrm{s}$, por lo que se le clasifica como Tipo C, según la FEMA (1994) y como S2: Suelo Firme, según la ATC (1985). Los valores de resistividad por lo general son mayores a los 40 ohmios-metro para rocas meteorizadas.

Tipo D (suelo blando): corresponde con los rellenos aluviales de la cuenca del Tempisque que forman suelos sedimentarios (Fig. 4). Los espesores de suelo en estos depósitos de relleno varían entre 10 - 60 m (SENARA, 1997). Los valores de velocidad de onda cortante registrados

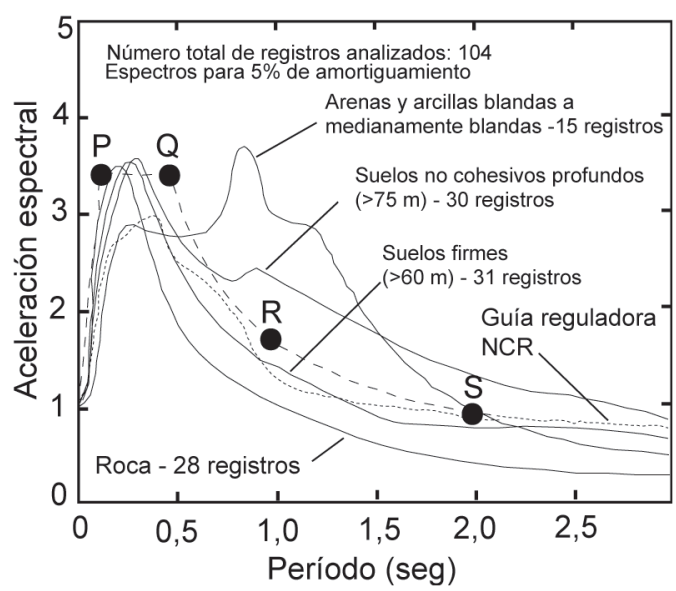

Fig. 3: Espectros para el $84,1 \%$ valor percentil para diferentes condiciones locales (Seed \& Idriss, 1982). en las capas de aluvión o suelo blando varían entre los $100-400 \mathrm{~m} / \mathrm{s}$, de lo cual se deduce que los suelos originados pueden tener valores inferiores a los $360 \mathrm{~m} / \mathrm{s}$, clasificando como Tipo D según la FEMA (1994) y como S3: Suelo Blando según la ATC (1985). Los valores de resistividad en éstos depósitos aluviales varían entre los 5 - 40 ohmios-metro, dependiendo de las proporciones de grava-arena-limo-arcilla, el grado de saturación y sales minerales en la matriz.

Tipo E (suelo con $V s<180 \mathrm{~m} / \mathrm{s}$ ): en esta clasificación entran los manglares o llanuras de inundación cuya clasificación según la ATC (1985) es como S4: Suelo Muy Blando.

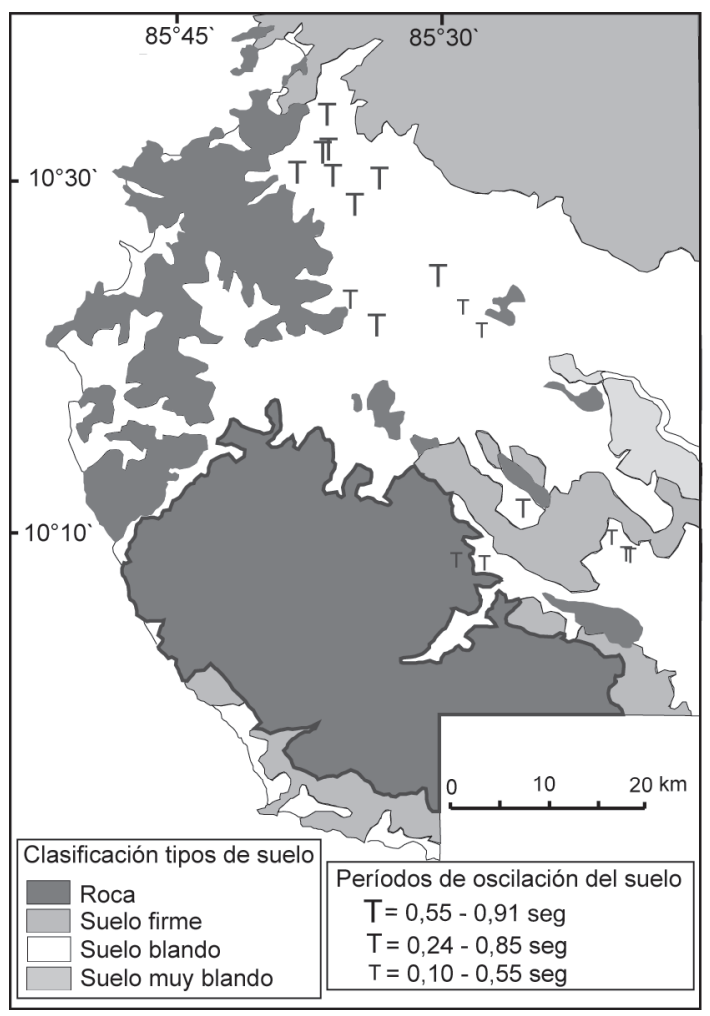

Fig. 4: Mapa de tipos de suelos para las diferentes condiciones de sitio y períodos de oscilación del suelo. Fuentes consultadas: Sáenz, R., 1982; ATC, 1985; FEMA, 1994; Funaioli \& Rossi, 1991; Denyer \& Arias, 1992 y 1993; Denyer et al., 1993; Arias, 1998; Tournon \& Alvarado, 1997; SENARA (base datos de pozos de 1999). 


\section{Determinación de los parámetros geofísicos}

Se consideraron cinco aspectos fundamentales para obtener los parámetros geofísicos de los perfiles de suelo y roca:

1. El tipo de litología presente (espesor y profundidad).

2. Consideraciones de los niveles freáticos (medio saturado o no saturado).

3. La variación de la velocidad de onda cortante con la profundidad.

4. Densidad relativa de cada capa geológica.

5. Determinación del módulo dinámico de corte.

La litología de cada perfil geológico se definió con base en los datos estratigráficos de los pozos y los registros de resistividad eléctrica de cada pozo. Los sondeos de resistividad eléctrica permitieron definir la profundidad del nivel freático en cada pozo y a la vez correlacionar las características litológicas para definir la presencia de capas de arcilla, limos, arena o grava, lo cual implica variaciones en los valores de velocidades de las ondas sísmicas compresiva y cortante y también, en los valores de densidad (Cuadro 2).

La consideración del nivel freático tiene importancia para evaluar la variación que tiene la velocidad de la onda compresiva en un medio saturado y no saturado. La onda compresiva, a diferencia de la onda cortante, puede propagarse en el agua, ésta generalmente aumenta su valor en medio saturado, debido a que los espacios o vacíos de los depósitos sedimentarios inconsolidados son ocupados por agua, lo cual también tiene implicaciones indirectas en los valores de la velocidad de la onda cortante en tanto se modifique

Cuadro 2

Parámetros geofísicos del perfil geológico del pozo CN-73 (NW de la hoja Carrillo Norte)

\begin{tabular}{|c|c|c|c|c|c|}
\hline \#capas & Litología & $\begin{array}{l}\text { Espesor } \\
(\mathrm{m})\end{array}$ & $\begin{array}{c}\text { PesoUnit. } \\
\left(\mathrm{kN} / \mathrm{m}^{3}\right)\end{array}$ & $\begin{array}{r}\text { G max } \\
(\mathrm{Mpa})\end{array}$ & $\begin{array}{c}\text { Vs } \\
(\mathrm{m} / \mathrm{s})\end{array}$ \\
\hline 1 & Arcilla plástica con poca grava & 4,6 & 14,4 & 33,0 & 150,0 \\
\hline 2 & Arcilla plástica con poca grava & 2,7 & 16,0 & 27,6 & 130,0 \\
\hline 3 & Arena media $(5-10 \%$ arcilla) & 0,9 & 16,8 & 55,5 & 180,0 \\
\hline 4 & Grava fina con matriz de arena gruesa y arcillosa $(30 \%)$ & 2,1 & 17,5 & 102,6 & 240,0 \\
\hline 5 & Sed. lacustres con arenas, arcillas y mat. volc. (arcillas 10-35\%) & 22,6 & 18,2 & 189,8 & 320,0 \\
\hline 6 & Arcilla muy plástica & 0,9 & 18,0 & 156,1 & 292,0 \\
\hline 7 & Sed. lacustres con arena y arcilla y mat. volc. & 2,7 & 18,3 & 216,1 & 340,0 \\
\hline 8 & Arenisca poco cementada & 1,8 & 18,4 & 229,8 & 350,0 \\
\hline 9 & Arcilla limosa con grava fina y lentes de arenisca (limo 40\%) & 6,4 & 18,6 & 266,3 & 375,0 \\
\hline 10 & Sed. lacustres con mat. volc. (arcillas $50 \%$ ) y lentes de arenisca & 2,7 & 18,7 & 292,7 & 392,0 \\
\hline 11 & Arena gruesa+grava con material volcánico (arcilla 5-10\%) & 1,8 & 18,7 & 305,7 & 400,0 \\
\hline 12 & Limos y arena limosa con poca grava y material volcánico & 3,7 & 19,0 & 393,2 & 450,0 \\
\hline 13 & Arena gruesa+grava fina con mat. volc. (limo $10 \%$, arcilla 10\%) & 2,7 & 19,2 & 431,4 & 470,0 \\
\hline 14 & Limo arcilloso con arena gruesa (arcilla $20 \%$ y arena $20 \%$ ) & 2,7 & 19,2 & 461,2 & 485,0 \\
\hline 15 & Basamento basáltico del Cretácico (Complejo de Nicoya) & - & 23,0 & 9904,3 & 2055,0 \\
\hline
\end{tabular}

Nota: El espesor máximo de suelo blando es $60 \mathrm{~m}$ y el nivel freático se localiza a 4,6 m de profundidad. Las características del perfil geológico como tipo de litología, espesor y nivel freático se obtiene mediante correlación litológica del pozo y su registro de resistividad eléctrica.

mat. volc.$=$ material volcámico, sed. $=$ sedimento 
la estructura o empaquetamiento del esqueleto del sedimento o depósito aluvial, o bien considerando el factor profundidad. Las velocidades de las ondas sísmicas compresiva y cortante generalmente aumentan con la profundidad debido a la compactación y rigidez del medio como resultado de esfuerzos litostáticos.

Se obtuvieron tres relaciones sobre la variación de las velocidades sísmicas de las ondas P y S con la profundidad en medio saturado y no saturado y aplicado a depósitos aluviales (gravas, arenas y arcillas). Para estas relaciones se utilizaron datos reportados en el área de estudio (Herrera, 1980; Leandro, 1975; Mora \& Protti, 1978; Protti, 1980; Protti, 1981 y Vásquez, 1985), mediciones realizadas en otras regiones de relleno aluvial, como Braja, 1993; Ishihara, 1996 y otras muchas publicaciones del Bulletin of the Seismological Society of America. Se les aplicó la regresión que mejor se ajustara a la dis-

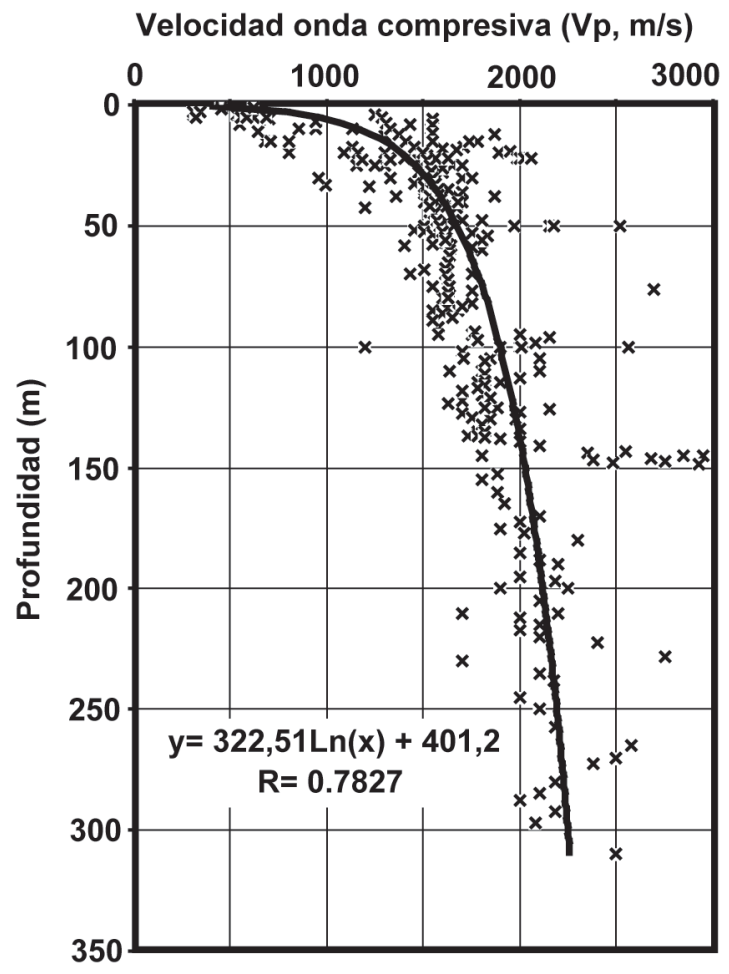

Fig. 5: Variación promedia de la velocidad de onda compresiva $\mathrm{P}$ con la profundidad en depósitos aluviales saturados y no saturados. persión de los datos para obtener el valor en las velocidades de onda $\mathrm{P}$ y $\mathrm{S}$ a cada profundidad (Figs. 5 y 6) o, dado el caso de tener el valor de velocidad de la onda $\mathrm{P}$, obtener por relación el valor de velocidad de la onda cortante S (Fig. 7).

Los valores de velocidad de la onda $\mathrm{P}$ en los depósitos aluviales se obtuvieron a partir de los resultados de refracción sísmica y resistividad eléctrica, obtenidos mediante los estudios de geofísica de varios informes técnicos del SENARA. Se registran valores en el rango de $700-1700 \mathrm{~m} / \mathrm{s}$ medidos a diferentes profundidades y en condiciones saturadas y no saturadas (Figs. 5 y 7). Se desarrolló una curva de velocidad de onda cortante $S$, en función de la profundidad y en condiciones saturadas (Fig. 6). Dichas correlaciones se generalizaron a depósitos aluviales sin tomar en cuenta específicamente si son arcillas, limos, arenas o grava, por cuatro factores:

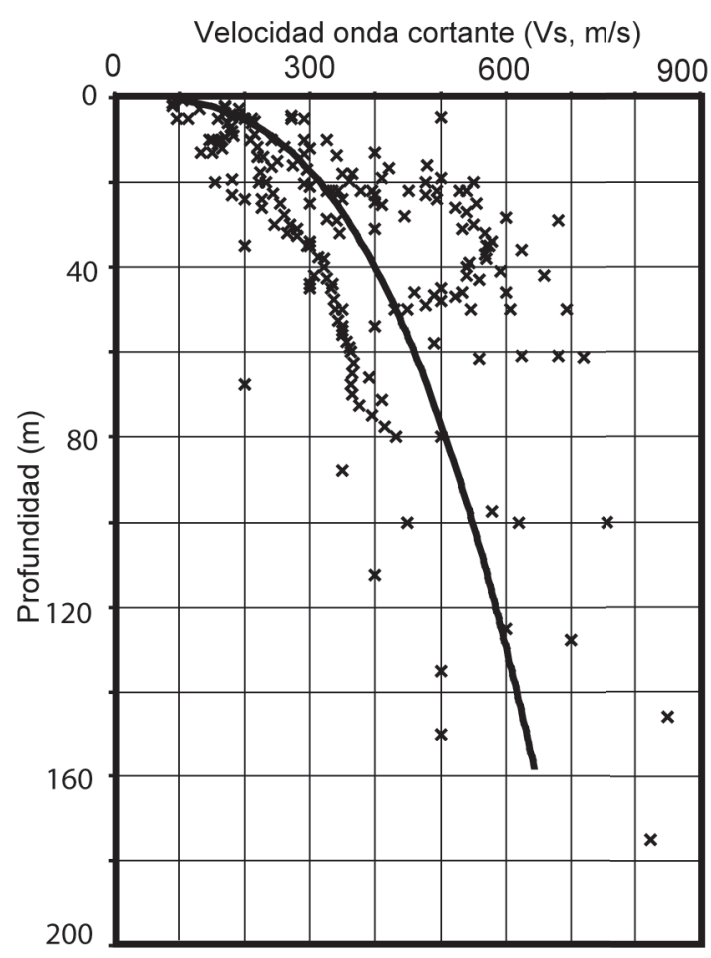

Fig. 6: Variación promedia de la velocidad de onda cortante $\mathrm{S}$ con la profundidad en depósitos aluviales saturados. 
1. La escala de trabajo (se cubre un área de estudio de 13 hojas cartográficas, Fig. 1).

2. La información de las fuentes (rellenos aluviales, estudios generalizados, falta de información detallada a depósitos de suelos).

3. La depositación de sedimentos en la cuenca del Tempisque es heterogénea (arcillas, limos, arenas y grava en mayor o menor proporción).

4. No se hizo geofísica exploratoria ni pruebas de laboratorio.

La figura 5 muestra la variación de la velocidad de la onda P con la profundidad. Esta relación incluye datos registrados específicamente en los depósitos aluviales al norte (hojas Belén y Carrillo Norte) y al suroeste (hojas Diriá y Villarreal). Se determinó que los datos registrados (27

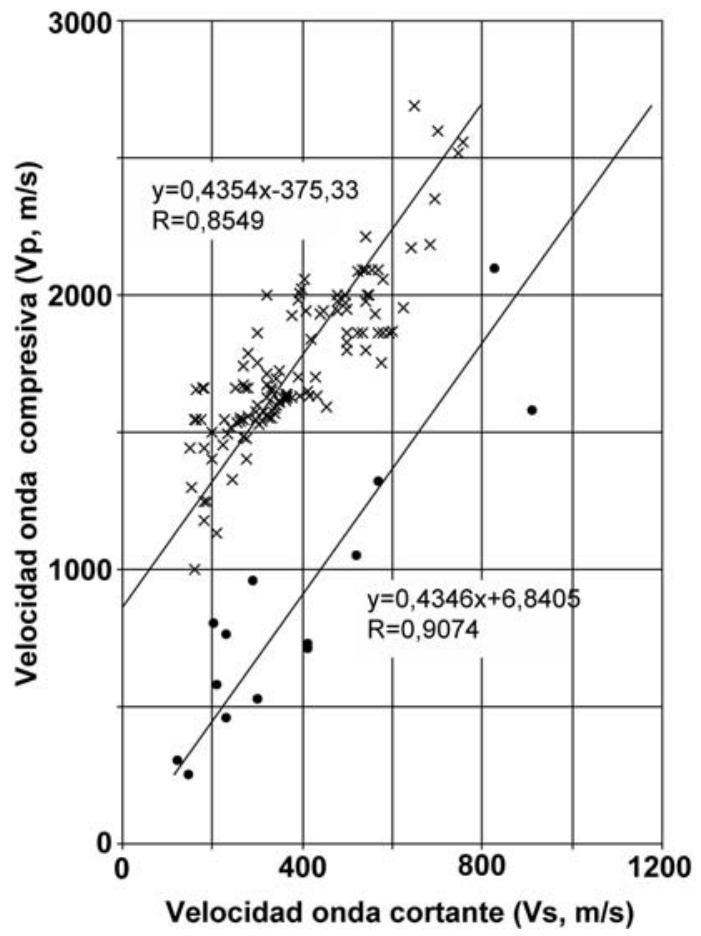

Fig. 7: Razón promedia entre las velocidades de onda cortante $\mathrm{S}$ y compresiva $\mathrm{S}$ en depósitos aluviales saturados y no saturados.

x Saturado $\quad$ No Saturado entre los 10-70 $\mathrm{m}$ de profundidad) presentan el mismo patrón de dispersión que las otras fuentes e inclusive algunos valores están dentro de la curva de regresión. Esta curva incluye valores tanto en medio saturado como no saturado. Analizando las correlaciones de datos se obtuvo que los valores en medio saturado se ubican a la derecha de la curva y los valores correspondientes al medio no saturado a la izquierda. Haciendo una evaluación de los valores con otras regiones de condiciones geológicas similares, se determinan tendencias muy similares para los primeros 50 metros de profundidad en la cuenca del Tempisque. Otro aspecto importante es la mayor variación que presentan los valores de velocidades de onda P entre los 5-70 m. Dicha curva empieza a ser funcional a partir de los $5 \mathrm{~m}$ de profundidad, debido a que existe desviación en los valores a profundidades menores.

La figura 6 muestra la variación de la velocidad de la onda cortante $\mathrm{S}$ con la profundidad en medio saturado. Se determinó que esta curva es funcional en depósitos de granulometría gruesa (arenas y gravas); sin embargo, puede ser práctica en depósitos de granulometría fina a profundidades más superficiales (0,2-2 metros). El valor de $\mathrm{R} 2=0,5506$, que muestra mayor dispersión en los datos relativos a las curvas dadas en las figuras 5 y 7 , puede ser explicado debido a que usualmente los valores en la velocidad de onda $\mathrm{S}$, se obtienen por métodos indirectos y son más difíciles de medir en relación con las velocidades de onda P. De ahí la importancia de analizar conjuntamente las variaciones de velocidades de onda $\mathrm{P}$ y $\mathrm{S}$ con la profundidad en medio saturado y no saturado, que se representa como la razón entre ambas en la figura 7. Esto nos lleva a un método de análisis para asignar los valores y variaciones en las velocidades de onda $\mathrm{S}$ en cada capa del modelo geológico de cada pozo.

La figura 7 muestra la relación entre las velocidades de las ondas $\mathrm{P}$ y $\mathrm{S}$ en medio saturado y no saturado. Se determinó que la regresión de la recta en medio saturado es aplicable en depósitos de granulometría fina y para valores de velocidad de la onda $P$ entre el rango 1300-2400 $\mathrm{m} / \mathrm{s}$. La regresión de la recta en medio no saturado es funcional en depósitos de granulometría 
gruesa (arenas y gravas) o en sedimentos más consolidados. Sin embargo, tiende a sobrestimar los valores de velocidad de la onda cortante S. En la regresión lineal de los datos en medio saturado se reporta un dato del área de estudio (2690/650), el cual es representativo de aluviones saturados en los alrededores de Nicoya.

Las correlaciones anteriores se utilizaron tomando en cuenta el rango de variación en la velocidad de onda $\mathrm{P}$ en gravas, arenas y arcillas. De esta forma se obtuvo un valor a cada profundidad, según la regresión aplicada y luego se procedió a definir el valor de velocidad de la onda S, trasladándose a uno u otro lado de la curva según el rango de variación para cada tipo de sedimento (granulometría, espesor y grado de saturación).

Una vez que se obtuvo la velocidad de la onda cortante $\mathrm{S}$, con la metodología anterior, se procedió a obtener el valor de densidad según la variación en los valores de la velocidad de la onda $\mathrm{S}$ a cada profundidad. Tomando en cuenta que la velocidad de la onda cortante varía con la rigidez del medio y el grado de saturación, se elaboraron dos curvas que relacionan la velocidad con la densidad en medio tanto saturado, como no saturado. Ambas regresiones permitieron obtener el valor de la densidad a cada profundidad según el valor de velocidad asignado (Figs. 8 y 9).

Al comparar ambas curvas para una velocidad de onda cortante dada, se puede observar que la curva correspondiente al medio no saturado presenta valores de densidad menor, comparados con la curva en medio saturado, en la cual aumenta significativamente (aprox.10\%) los valores de densidad, debido a la presencia de agua en los poros. El agua no presenta resistencia al cortante, cuyo efecto es el de la onda S, por tanto es de esperar que la velocidad de la onda cortante no tenga una variación significativa si comparamos los valores en medio saturado y no saturado, a menos que la presencia de agua o saturación en los sedimentos inconsolidados modifiquen significativamente la estructura o empaquetamiento de los depósitos de aluvión o suelo.

Una vez que se tiene la velocidad de onda cortante y la densidad (dado en peso unitario, $\mathrm{kN} / \mathrm{m}^{3}$ ), se calcula el módulo dinámico de corte (G) en MPa, según la relación:

$$
G_{\max }=\rho \bullet V_{s}^{2}
$$

donde:

$\rho$ : es la densidad en $\mathrm{kg} / \mathrm{m}^{3}$

Vs: es la velocidad de la onda cortante en $\mathrm{m} / \mathrm{s}$

Los estudios de refracción sísmica en el área, establecen varios rangos en la velocidad de la onda P para roca. Estas velocidades corresponden a las rocas del Complejo de Nicoya, que se consideran como el basamento de la región. Dichas rocas son generalmente basaltos e intrusivos y en algunos casos rocas silíceas sedimentarias.

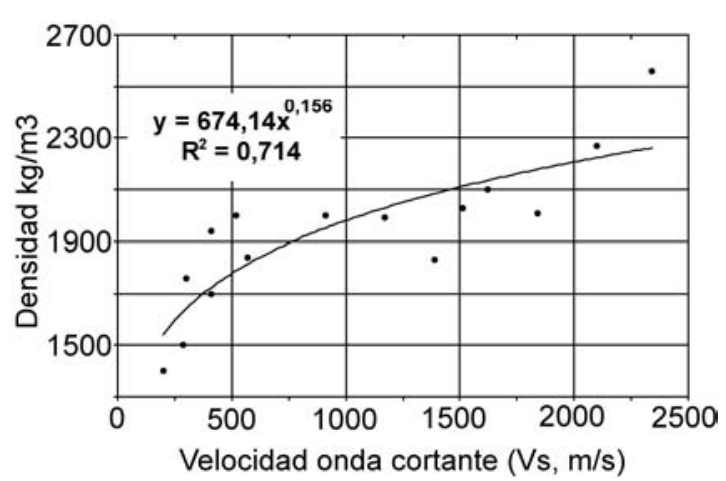

Fig. 8: Variación promedia de la densidad con la velocidad de onda cortante en depósitos aluviales no saturados.

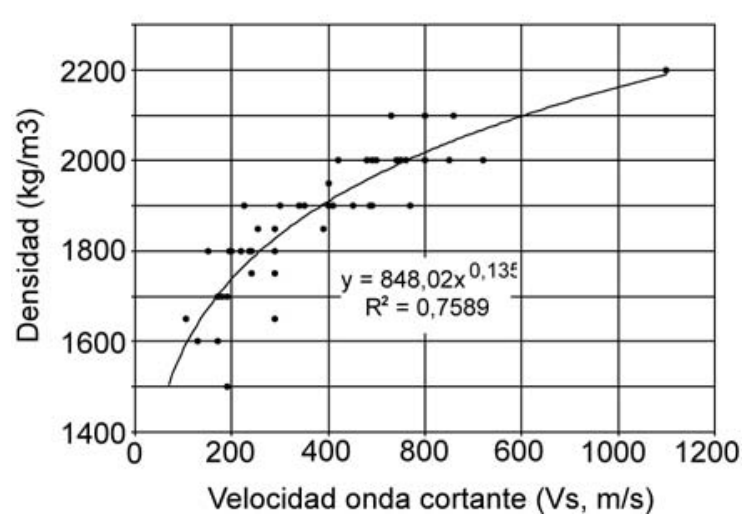

Fig. 9: Variación promedia de la densidad con la velocidad de onda cortante en depósitos aluviales saturados. 
Se reportan valores entre el rango $2700-5800 \mathrm{~m} / \mathrm{s}$ en la cuenca del río Andamojo al suroeste del área (Protti, 1980), 3030-4860 m/s en la margen derecha del río Tempisque (S de Filadelfia y W de Belén), San Blas y Sardinal (Protti, 1981), $2700-3560 \mathrm{~m} / \mathrm{s}$ al SW de Filadelfia (Vásquez, 1985). Considerando estos rangos en la velocidad de onda $\mathrm{P}$, se procedió a tomar un valor promedio en cada caso y asignar el valor correspondiente en cada pozo según su cercanía al lugar donde se reportan los datos de velocidad en los estudios de refracción sísmica. Para tomar el valor correspondiente de velocidad de la onda $S$, se utilizó la siguiente relación que generalmente se aplica en sismología para roca:

$$
V_{p}=\sqrt{3 V_{s}}
$$

También se utilizó una curva de correlación entre las velocidades sísmicas $\mathrm{P}$ y $\mathrm{S}$ para asignar valores de velocidad de onda cortante en roca para cada pozo. La densidad en roca se obtuvo relacionando la velocidad de la onda $\mathrm{S}$ con la densidad.

\section{MÉTODOS ESPECTRALES Y FUNCIONES DE TRANSFERENCIA}

Los espectros de respuesta de terremotos reales son altamente irregulares, sus formas reflejan los detalles de sus contenidos de frecuencia específicos y fase. Existen varios aspectos que tienen influencia en las características del espectro de respuesta: la magnitud, el mecanismo de ruptura de la falla y la distancia entre la estación y el epicentro son los más importantes.

Los espectros de respuesta describen la respuesta máxima de un sistema de un grado de libertad ante una excitación sísmica o la historia de aceleraciones dada, como una función de la frecuencia natural y amortiguamiento propios del sistema. Por lo tanto, la forma de los espectros de respuesta indican que los valores máximos de aceleración, velocidad y desplazamiento están asociados a rangos de frecuencia diferentes. Por eso estos se dividen generalmente en tres zonas: zona de dominio de la aceleración (frecuencias altas), zona de dominio de la velocidad (frecuencias intermedias) y zona de dominio del desplazamiento (frecuencias bajas). Dado que el espectro representa el movimiento en una componente, lo usual es evaluar éste, en una región determinada a partir de un registro de aceleración del terreno, tomando la componente horizontal de mayor amplitud que representa el movimiento de entrada en el programa.

Los depósitos de suelos blandos (aluviales) se comportan como un filtro que atenúa o amplifica las diferentes frecuencias de las ondas sísmicas que forman la señal original. Este proceso de filtrado o transformación de la señal sísmica original y su importancia en el comportamiento de las estructuras se visualiza mejor, cuando se utiliza el espectro de respuesta del registro obtenido en la superficie del terreno. Este comportamiento se puede representar mediante una función de tipo espectral (función que depende de la frecuencia o el período), que se denomina generalmente como espectro de amplificación del depósito de suelo en particular, o función de transferencia, que toma la señal base y la transforma a medida que viaja hacia la superficie. Es importante observar en este sentido, que la señal en suelo se amplifica para los períodos largos con respecto a la respuesta en roca (Figs. 2 y 3 ).

La función de transferencia se define como la razón entre la amplitud del movimiento del suelo en la superficie respecto a la amplitud en roca aflorante y se supone que es característica de cada sitio. Permite determinar a cuales frecuencias o períodos se producen las mayores amplificaciones en el terreno. Los factores de amplificación obtenidos de dichas funciones se aplican a los parámetros de aceleración, velocidad y desplazamiento.

\section{RESULTADOS DE LOS TRES GRUPOS DE POZOS ANALIZADOS}

Los resultados de los tres grupos de pozos se presentan en términos de aceleración espectral, correlacionado con los resultados de las funciones de transferencia. La importancia de las 
funciones de transferencia transciende en el hecho de que constituyen una forma de corroborar los datos espectrales, dado que en principio los resultados de éstas dependen más de las características del perfil geológico que del movimiento sísmico de entrada utilizado.

El Cuadro 1 resume los resultados generales que se obtienen de los tres grupos de pozos analizados. Se toman en cuenta los valores de períodos de mayor amplificación, los rangos de período y el período de mayor amplificación utilizando los valores espectrales y las funciones de transferencia con ambos sismos. Los resultados se enfocan en determinar los rangos de período y el período de oscilación del suelo en el cual ocurre la máxima amplitud, mas que en determinar la magnitud del parámetro amplificado (aceleración) debido a que se ha observado que en algunos casos el programa tiende a sobrestimar los valores de amplificación.

Para el primer grupo de pozos se obtiene un rango de períodos entre 0,55-0,91 s donde ocurre amplificación y con los mayores espesores de suelo blando entre 35-60 m (Cuadro 1). Se tienen ocho períodos importantes de amplificación en orden de importancia dados en 0,$85 ; 0,55$; 0,$65 ; 0,24 ; 0,32 ; 0,14 ; 0,26$ y $0,18 \mathrm{~s}$, siendo los más importantes en 0,88 y 0,71 s correlacionando los valores espectrales y las funciones de transferencia en ambos sismos (Fig. 10 y Cuadro 1).

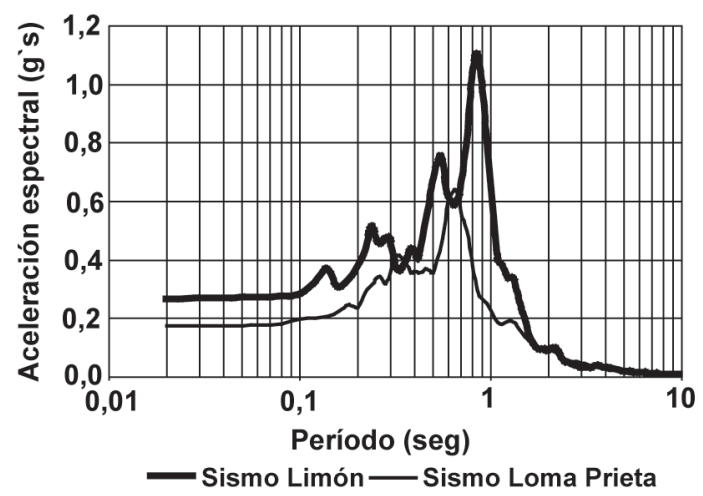

Fig. 10: Espectros de respuesta promedio de las aceleraciones (primer grupo de pozos).
Para el segundo grupo de pozos se tiene un rango entre $0,24-0,85 \mathrm{~s}$, con espesores intermedios de suelo blando entre 30-50 m (Cuadro 1). Se tienen siete períodos de amplificación importantes dados en 0,$55 ; 0,85 ; 0,46 ; 0,24 ; 0,65$; 0,14 y $0,18 \mathrm{~s}$, siendo los más importantes en 0,65 y 0,54 s correlacionando los valores espectrales y las funciones de transferencia en ambos sismos (Fig. 11 y Cuadro 1).

Para el tercer grupo de pozos se tiene un rango entre $0,10-0,55 \mathrm{~s}$ donde ocurre la amplificación máxima y con los espesores mínimos de suelo blando entre los 10-20 m (Cuadro 1). Para el grupo A se tienen ocho períodos de mayor amplificación en orden de importancia dados en 0,$24 ; 0,1 ; 0,55 ; 0,26 ; 0,18 ; 0,32 ; 0,85$ y $0,65 \mathrm{~s}$, siendo el más importante en $0,23 \mathrm{~s}$ de igual forma correlacionando los valores espectrales y las funciones de transferencia en ambos sismos (Fig. 12a y Cuadro 1). Para el grupo B se tienen seis períodos de mayor amplificación dados en 0,14 ; 0,$12 ; 0,24 ; 0,55 ; 0,65$ y 0,85 , siendo el más importante en $0,14 \mathrm{~s}$ (Fig. 12b y Cuadro 1)

La Fig. 4 muestra el mapa con la distribución de los resultados de períodos de oscilación del suelo para los valores críticos de amplificación, obtenidos a partir de las correlaciones de los valores espectrales y las funciones de transferencia según el Cuadro 1. Del mapa se observa, que los períodos de oscilación del suelo se dan en

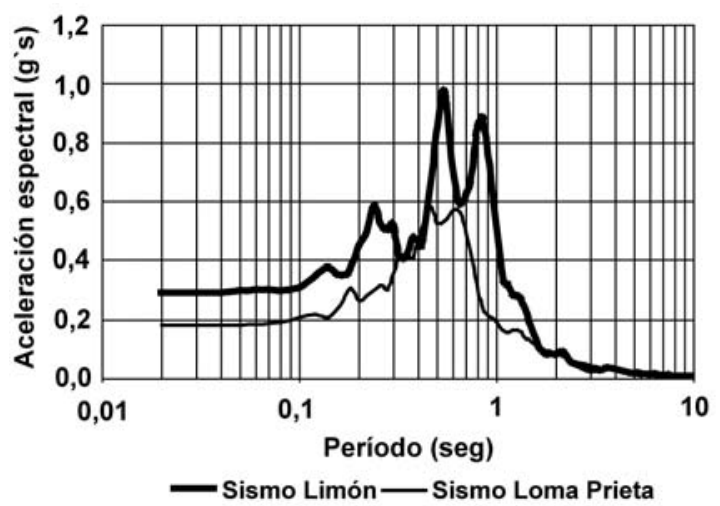

Fig. 11: Espectros de respuesta promedio de las aceleraciones (segundo grupo de pozos). 
el rango entre $0,55-0,91 \mathrm{~s}$ en la región noroeste (primer grupo de pozos). Los valores de períodos intermedios $(0,24-0,85 \mathrm{~s})$ y mínimos $(0,10-$ $0,55 \mathrm{~s})$ se presentan en la región central - sureste, correspondiendo con las áreas del segundo y tercer grupo de pozos.

\section{DISCUSIÓN Y CONCLUSIONES}

El escenario geológico donde ocurren los sismos de subducción frente a la península de Nicoya se dan generalmente hacia el borde de la costa pacífica, cuyo proceso de ruptura de fallamiento inverso favorece un patrón de radiación de la energía hacia el océano (Kelleher et al., 1973). Los resultados del programa indican que las mayores amplificaciones se dan en rangos de frecuencia similares a los contenidos en los movimientos de entrada utilizados. Si comparamos las frecuencias predominantes dadas en los espectros de Fourier de las aceleraciones en los dos movimientos de entrada utilizados y los resultados de las frecuencias predominantes dadas en los espectros de Fourier de las aceleraciones con ambos sismos para los pozos analizados, notamos que existe buena correlación con los resultados de la respuesta espectral y las funciones de transferencia (Rojas, 1998). Es importante la similitud que existe en los resultados obtenidos con las funcio-

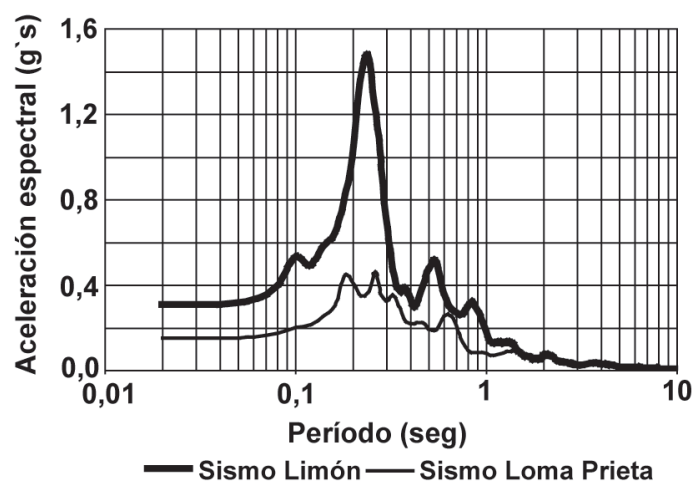

Fig. 12a: Espectros de respuesta promedio de las aceleraciones (tercer grupo de pozos). nes de transferencia para el sismo de Loma Prieta en relación con la respuesta espectral del sismo de Limón para el período de mayor amplificación (Cuadro 1). A pesar de que son sismos ocurridos en escenarios geológicos diferentes, existe buena correlación en los períodos de máxima amplitud. Sin embargo, los factores de amplificación son diferentes y por lo tanto los valores de amplificación dan resultados diferentes. Las diferencias en los valores de amplificación se puede explicar debido a que el nivel energético de cada sismo es diferente, al comparar los espectros de potencia de las aceleraciones en ambos sismos (Rojas, 1998). Los espectros muestran que el nivel energético del sismo de Limón es 1,84 veces mayor que el sismo de Loma Prieta para la máxima amplitud. Los sismos de subducción registrados a larga distancia tienden a tener contenido de frecuencias bajas dependiendo de la distancia de la fuente al sitio donde es registrado. Dado que los dos sismos utilizados no se dieron en un escenario de subducción sería de esperar que los resultados del programa difiera para una situación real.

Un aspecto importante en el análisis de resultados es que los períodos de mayor amplificación dados en 0,$85 ; 0,55 ; 0,24$ y 0,14 s para el sismo de Limón y 0,65 y 0,18 s para el sismo de Loma Prieta son característicos como períodos de mayor amplitud en ambos sismos. Esto es que los registros sintéticos son muy dependientes del

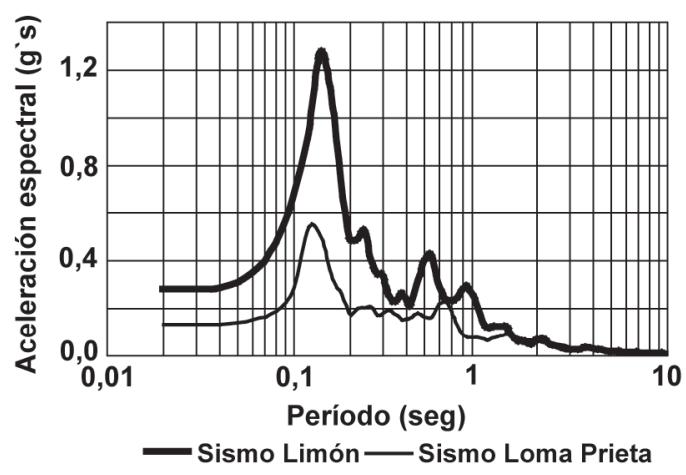

Fig. 12b: Espectros de respuesta promedio de las aceleraciones (tercer grupo de pozos). 
registro del movimiento introducido. La conclusión importante es que el período de oscilación del suelo disminuye con el espesor de suelo blando, lo cual es independiente del registro de entrada utilizado. Para el caso particular del área de estudio, los resultados dan una variación del período de oscilación del suelo de $0,88 \mathrm{~s}$ hasta valores mínimos de 0,14 s en dirección NW-SE, paralelo al eje del valle de la cuenca del Tempisque. Por tanto, las oscilaciones del terreno pueden darse en el rango de frecuencias de $1-7 \mathrm{~Hz}$.

Tales variaciones en los valores y rangos de períodos de oscilación del suelo obtenidos en los tres grupos de pozos y ubicados en tres áreas diferentes están dados por tres factores fundamentales: cambio en el espesor del suelo blando con valores máximos de $60 \mathrm{~m}$ hasta valores mínimos de $10 \mathrm{~m}$ en dirección NW-SE; la condición de roca sedimentaria como basamento del suelo blando, parcialmente en el área del segundo grupo y totalmente en el área del tercer grupo y cambios físicos y geofísicos de las propiedades del suelo blando, según el modelo de capas geológicas analizado en cada uno de los grupos de pozos analizados. Los cuales son dados por diferencias genéticas y diagénesis de los sedimentos derivados de las formaciones geológicas adyacentes.

Los resultados de aceleraciones máximas para el primer grupo de pozos se dan en el rango de períodos entre $0,55-0,91 \mathrm{~s}$ para un período fundamental de oscilación del suelo aproximado en 0,88 y $0,71 \mathrm{~s}$. En esta área se ubican los mayores espesores de suelo blando, con valores entre los 35-60 m.

Para el segundo grupo se dan en el rango entre $0,24-0,85 \mathrm{~s}$, para un período fundamental de oscilación aproximado en 0,65 y 0,54 s. Se presentan los espesores intermedios con valores entre los 30-50 m.

El tercer grupo presenta valores máximos en el rango de 0,10-0,55 s, presentándose dos períodos fundamentales de oscilación del suelo en 0,23 y 0,14 s. Para este grupo los períodos de mayor amplificación de las funciones de transferencia se correlacionan muy bien con los valores espectrales. Es importante mencionar que estos períodos se presentan también como períodos importantes en los dos movimientos de entrada utilizados. En este grupo se presentan los mayores factores de amplificación, tanto que sobrestiman los valores para las amplitudes máximas. En el área de este grupo se presentan los espesores mínimos menores a los 20 m con excepción del pozo TAL-122 que es mayor a los $30 \mathrm{~m}$.

El resultado general de que el período fundamental de oscilación del suelo disminuye con la disminución del espesor del suelo blando parece ser una conclusión evidente, acorde con los estudios de Seed \& Idriss (1982).

Los valores espectrales de amplificación en los rangos de períodos determinados se dan entre $0,5-1,1 \mathrm{~g}$ para el caso del primer grupo de pozos; 0,55-0,98 g para el segundo grupo; 0,41,5 g para el caso del tercer grupo de pozos. Los factores de amplificación para el tercer grupo tienden a sobrestimar los valores máximos de amplificación con valores de hasta $2 \mathrm{~g}$.

Según los principios básicos del programa SHAKE, el análisis debe ser aplicado a perfiles geológicos ubicados en las partes centrales de las cuencas sedimentarias, para evitar los efectos laterales como acuñamientos e interdigitaciones de los depósitos sedimentarios, así también como las reflexiones y refracciones de las ondas sísmicas debido a las irregularidades de los límites inferiores de las cuencas. Se consideró importante el hecho de que los grupos de pozos analizados sean paralelos al rumbo de lo que se considera el eje del valle sinclinal o graben del la cuenca del Tempisque, donde se ubican los mayores espesores de aluvión, por lo cual se minimizan los efectos laterales del valle sedimentario y las irregularidades del límite inferior de la cuenca.

Según los resultados los valores de períodos obtenidos con las funciones de transferencia comparados con los valores de períodos espectrales, se obtienen en rangos de frecuencia que pueden ser aplicados mejor en el dominio de las aceleraciones en cada grupo de pozos. Lo anterior debido a que, por lo general se observa que los períodos de amplificación en las funciones de transferencia para los segundos y terceros valores son menores que los valores espectrales en cada 
grupo de estudio. De tal forma, que los valores de períodos mayores puedan ser aplicados en el dominio de las velocidades.

Según los criterios de la FEMA los suelos en el área de estudio se pueden clasificar en cuatro tipos: B, C, D y E (roca, suelo muy denso y roca blanda, suelo blando, suelo muy blando) respectivamente. La clasificación anterior se hizo correlacionando criterios litológicos de las formaciones geológicas y propiedades geofísicas (velocidad de onda cortante, resistividad, densidad) que en algunos casos se infieren o son generalizados debido a la escala de trabajo.

La metodología y la investigación permite obtener una aproximación de las áreas con amenaza de amplificación sísmica y los principales factores involucrados. También identifica áreas donde se debe de tomar en consideración las características de las formaciones geológicas superficiales (suelos rocosos, firme, blando y muy blando) y ayuda a definir factores de amplificación dinámica, para perfiles de suelo blando. También permite orientar recursos económicos y estudios geológicos, geotécnicos y geofísicos para el desarrollo de infraestructura civil. Es importante la determinación de las propiedades in situu, desde el punto de vista geofísico de la geología superficial para la evaluación de obras críticas. Sin embargo, la metodología permite obtener una correlación con sitios de condiciones geológicas similares, para una aproximación de los resultados.

\section{REFERENCIAS}

APLIED TECHNOLOGY COUNCIL, 1985: Earthquake damage evaluation data for California. - 492 págs. ATC - 13, Redwood.

ARIAS, M., 1998: Interpretación geomagnética de los proyectos geotérmicos de Tenorio, Miravalles y Rincón de la Vieja, cordillera de Guanacaste, Costa Rica. - 129 págs. Univ. de Costa Rica, San José [Tesis Lic].
BRAJA, M. DAS, 1993: Principles of soil dynamics. - 570 págs. PWS-KENT Publ. Comp. Boston.

DENYER, P. \& ARIAS, O., 1992: Mapa Geológico de las hojas Belén, Carrillo Norte, Matapalo y Punta Gorda, Guanacaste, Costa Rica. - Escala 1:50000, Rev. Geol. América Central, 16.

DENYER, P., ARIAS, O. \& HERNÁNDEZ, J., 1993: Estructura geológica cuaternaria del norte y centro de la península de Nicoya, Guanacaste, Costa Rica. - 23 págs. Univ. Costa Rica, Proyecto Nicoya.

DENYER \& ARIAS, 1993: Geología del norte de la península de Nicoya, Costa Rica. Rev. Geol. América Central, 16: 69-84.

PROSHAKE 1998: Ground analysis program (Version 1.0). User's Manual. - 55 págs. EduPro Civil Systems, Inc. Redmond, Washington.

FEDERAL EMERGENCY MANAGEMENT AGENCY, 1994: NEHRP recommended provisions for seismic regulations for new buildings. FEMA. Part 1. -7 págs.

FUNAIOLI, G. \& ROSSI, L., 1991: IL complesso volcanico de Rincón de la Vieja (Costa Rica): Evoluzione geo-volcanologica e chimica-petrografico. - 207 págs. Univ. Pisa, Facolta Scienze Matematiche, Fisiche e Natural, [Tesis Laurea].

GALLEGOS, R., 1980: Estudio de un modelo para la evaluación sísmica local de los depósitos de suelo. - 284 págs. Univ. Costa Rica [Tesis Lic.].

HERRERA, J., 1980: Hidrogeología de la cuenca del río Andamojo, Santa Cruz, Guanacaste, Costa Rica. - 53 págs. Informe téc. 132. Proyecto Península de Nicoya SENAS (SENARA). 
ISHIHARA, K., 1996: Soil behaviour in earthquake geotechnics. -350 págs. Clarendon Press, Oxford.

KELLEHER, J., SYKES, L. \& OLIVER, J., 1973: Possible criteria for predicting earthquake locations and their application to major plate boundaries of the Pacific and the Caribbean. - J. Geoph. Res. 78(14).

LEANDRO, G., 1975: Informe del estudio preliminar efectuado con resistividad eléctrica en el área de San Antonio de Nicoya. - 35 págs. Informe téc. 87, abril 1975. SENAS (SENARA).

MORA, D. \& PROTTI, R., 1978: Geología regional del sitio de la represa derivadora del río Magdalena Cañas, Guanacaste. 21 págs. Informe téc.117, SENAS (SENARA).

PROTTI, R, 1980: Prospección geofísica por sísmica de refracción en la cuenca del río Andamojo, Santa Cruz, Guanacaste, con fines hidrogeológicos. 38 págs. Informe téc.133, SENAS (SENARA).

PROTTI, R., 1981: Estudio hidrogeológico con fines de riego en la margen derecha del río Tempisque. -47 págs. Informe téc. 152 de geofísica (más anexo). Departamento de Hidrogeología, SENAS (SENARA).

PUJOL, R. \& CASTRO, L., 1981: Propiedades dinámicas de los suelos de San José y Cartago. - Seminario de Ingeniería Estructural, 29, 30 y 31 de octubre, San José, Costa Rica: 95-109.

RAMÍREZ, R., SANTANA, G. \& CHACÓN, O., 1994: Mapa de amplificación sísmica del Valle Central, Costa Rica. IX Congr.
Intern. - 23 págs. Pronóstico de Terremotos. Hotel Herradura, setiembre 1994.

RAMÍREZ, R., 1995: Microzonificación sísmica de San Ramón, Alajuela, Costa Rica. 200 págs. Univ. Costa Rica, San José [Tesis Lic.].

ROJAS, M., 1998: Amplificación de la señal sísmica en depósitos aluviales de la Península de Nicoya, Costa Rica. - 147 págs. Univ. Costa Rica, San José [Tesis Lic.].

SÁENZ, R., (Coord.) 1982: Mapa Geológico de Costa Rica, escala 1:200 000 (hojas Nicoya y Liberia). - Ministerio de Industria Energía y Minas. Dirección de Geología, Minas y Petróleo. Instituto Geográfico Nacional de Costa Rica.

SEED, H.B. \& IDRISS, I.M., 1982: Ground motions and soil liquefaction during earthquakes. - 134 págs. Earthquake Engineering Research Institute (EERI), Pasadena, California.

SENARA, 1997: Base de datos de pozos. Archivo de pozos. Hojas Villarreal (VI 1 - VI 14); Garza (GR 1 - GR 40); Matapalo y Punta Gorda (MTP 1 - MTP 170); Talolinga (TAL 1 - TAL 200); Tempisque (TE 1, TE 9, TE 25, TE 26 - TE 78); Cerro Brujo (CJ 1 - CJ 4); Matambú (PMT 1 - PMT 200).

TAYLOR, W., 1994: Características sismo-dinámicas del suelo blando en la región central de la ciudad de Cartago, Costa Rica. 100 págs. Univ. Costa Rica, San José [Tesis Lic.].

TOURNON, J. \& ALVARADO, G., 1997: Mapa Geológico de Costa Rica 1:500000. Folleto explicativo. - 79 págs. Ministère des Affaires Ètrangères (Délégation 
Régionale à la Coopération Scientifique et Technique); Instituto Costarricense de Electricidad (ICE). Ed. Tecnología de Costa Rica.

VALVERDE, M.A., 1981: Análisis de la respuesta dinámica de depósitos de suelo ubicados en varias ciudades del Valle Central. 221 págs. Esc. Ingeniería Civil, Univ. Costa Rica [Tesis Lic.].
VARGAS, W.J., 1987: Análisis dinámico de depósitos de suelo. - 87 págs. INII. Lab. Ingeniería Sísmica, UCR. Reporte INI, 41.

VÁSQUEZ, C., 1985: Zonificación hidrogeológica por métodos geofísicos de las Cooperativas del Río Las Palmas. - 32 págs. Informe téc. 158 (Dirección de Aguas Subterráneas y Estudios Básicos, Departamento de Hidrogeología). SENARA. 